\title{
Smartphone-assisted endoscopic surgery via Kocher's point for intraventricular hemorrhage caused by thalamic hemorrhage: A comparison with external ventricular drainage
}

\author{
XIN GE ${ }^{1-3^{*}}$, XINHUA XU ${ }^{1 *}$, XINGUANG YU ${ }^{1}$, XIAOLEI CHEN ${ }^{1}$, DUO LI $^{2}$, YANHUA XU $^{2,3}$ and YUE ZHANG ${ }^{2,3}$ \\ ${ }^{1}$ Department of Neurosurgery, Chinese PLA General Hospital, Beijing 100853; \\ Departments of ${ }^{2}$ Neurosurgery and ${ }^{3}$ Neuro-ICU, Central Hospital of Jinzhou, Jinzhou, Liaoning 121001, P.R. China
}

Received August 25, 2018; Accepted May 23, 2019

DOI: $10.3892 /$ etm. 2019.7740

\begin{abstract}
The aim of the present study was to investigate the efficacy of endoscopic surgery via a Kocher point approach for intraventricular hemorrhage (IVH) caused by thalamic hemorrhage (TH), in comparison with external ventricular drainage (EVD). Between January 2016 and August 2017, a total of 40 patients with IVH caused by TH were enrolled. The patients were assigned to an EVD group and an endoscopic surgery group in a non-randomized manner. The pre-operative characteristics recorded included age, pre-operative Glasgow Coma Scale (GCS) score, hemorrhage volume, length of neuro-intensive care unit stay and Graeb score of IVH. Outcomes included the GCS score at 2 weeks after surgery, 30-day and 90-day mortality rates, modified Rankin scale at 6 months after ictus and ventriculo-peritoneal (VP) shunt rate. The baseline characteristics were not significantly different between the two groups. The VP shunt rate in the EVD group was significantly higher compared with that in the endoscopic surgery group (50.0 vs. $15.0 \%$; $\mathrm{P}=0.02$; odds ratio, 5.7$)$. In conclusion, smartphone-assisted endoscopic surgery via Kocher's point is feasible and safe, and significantly reduces permanent shunt dependency for IVH caused by TH.
\end{abstract}

\section{Introduction}

Spontaneous intracerebral hemorrhage (ICH) is a type of neurosurgical emergency, which is particularly common in East Asia due to ethnic backgrounds and eating habits (1-3).

Correspondence to: Professor Xinguang Yu, Department of Neurosurgery, Chinese PLA General Hospital, 28 Fuxing Road, Beijing 100853, P.R. China

E-mail: yuxinguang301@126.com

\section{${ }^{*}$ Contributed equally}

Key words: endoscopic surgery, external ventricular drainage, intraventricular hemorrhage, thalamic hemorrhage, smartphoneassisted surgery
Thalamic hemorrhage (TH) accounts for $30 \%$ of all cases of ICH $(4,5)$. TH is frequently accompanied by intraventricular hemorrhage (IVH), which is associated with high mortality and morbidity rates due to a high incidence of complications, including obstructive hydrocephalus, hypothalamic storm or hyperthermia. In recent years, an increasing number of studies have demonstrated that evacuation of hematoma in the ventricle is associated with a better prognosis $(1-3,5,6)$. The major aim of surgery is to evacuate the hematoma maximally, while minimizing injury to normal brain tissues. Therefore, it is necessary to use a navigation tool to precisely devise the trajectory of surgery. In a previous study, our group demonstrated the feasibility of hematoma evacuation using a Kocher's point approach under the assistance of smartphone navigation and 3D Slicer image reconstruction (7). The aim of the present study was to investigate the efficacy of endoscopic surgery via a Kocher's point approach for IVH caused by TH, in comparison with external ventricular drainage (EVD).

\section{Materials and methods}

Patients. A contemporary, non-randomized, controlled design was adopted for the present study. From January 2016 to August 2017, 64 patients diagnosed with TH were treated at the Departments of Neurosurgery and Neuro-Intensive Care Unit (NICU), Central Hospital of Jinzhou (Jinzhou, China). Patients with IVH caused by TH who had a Glasgow Coma Scale (GCS) score (8) of $\leq 12$ were indicated for surgery. Finally, 40 patients were included in the study and were allocated to an EVD group or an endoscopic surgery group. As the patients were in comas, the decision of what treatment the patient would receive was made by the legally authorized representative. The inclusion criteria were as follows: i) Hypertensive TH with IVH confirmed by computed tomography (CT) scan; ii) hematoma volume $\geq 25 \mathrm{ml}(7,9,10)$; iii) patient age $\leq 75$ years; iv) $4 \leq \mathrm{GCS} \leq 12$; v) written informed consent provided by the patient's legally authorized representative. The exclusion criteria were as follows: i) Secondary hemorrhage caused by trauma, aneurysms, vascular malformations or tumor; ii) multifocal bleeding with the exception of extension of TH to another location; iii) brainstem failure indicated by bilateral mydriasis, decerebrate rigidity and unstable vital signs; iv) severe dysfunction of the 
heart, liver or kidney; v) mortality within 3 days (7). The present study was approved by Ethics Committee of Central Hospital of Jinzhou (Jinzhou, China) and was performed in conformity to the Declaration of Helsinki. All patient's legally authorized representative provided written informed consent prior to enrolment in the present study.

Surgery. In the EVD group, standard EVD was performed on patients under local anesthesia and intravenous sedation (11). No intraventricular injection of thrombolytics (e.g. urokinase) or aspiration were performed during the surgical procedure. At $6 \mathrm{~h}$ after EVD, 20,000 units of urokinase were injected through the catheter to dissolve the residual hematoma and facilitate drainage. The drainage bottle was then opened but elevated to the height of $27 \mathrm{~cm} \mathrm{H}_{2} \mathrm{O}$ for $2 \mathrm{~h}$ to allow for drug and hematoma interaction. This prevented high intracranial pressure and undetected obstructive hydrocephalus. After $2 \mathrm{~h}$, the drainage bottle was reduced to $15 \mathrm{~cm} \mathrm{H}_{2} \mathrm{O}$ for drainage. Injection of urokinase was performed twice a day for 3-5 days. Patients with ventricular blood received continuous drainage at $15 \mathrm{~cm} \mathrm{H}_{2} \mathrm{O}$ until there was no further reduction in cerebrospinal fluid (CSF) blood content. The EVD catheter was then elevated to the height of $27 \mathrm{~cm} \mathrm{H}_{2} \mathrm{O}$. The catheter was removed if the drainage volume was $<50 \mathrm{ml}$ over the next $24 \mathrm{~h}$. Patients were considered as having failed catheter 'elevation' if they developed hydrocephalus or their consciousness deteriorated.

In the endoscopic surgery group, smartphone-assisted navigation was used (Fig. 1). First, four fiducial markers (electrode pads) were attached to the patient's head at the following locations: Nasion (F-1), external auditory canal on both sides (F-2, F-3) and entry point (Kocher's point). The fiducial F-1, F-2 and F-3 were marked to define the first reference plane. The reference fiducials F-4 and F-5 were indicated on the midline of the CT image, usually on the cerebral falx. Using these two fiducials, the second reference plane was then defined and the third reference plane was automatically generated. The entry point was $1 \mathrm{~cm}$ anterior to the coronal suture and $2.5 \mathrm{~cm}$ lateral to the midline. This was named Kocher's point and selected for endoscopic port insertion. The target point was the bottom of the thalamic hematoma rather than the IVH hematoma, and was marked to define the trajectory. After removing the hematoma of the thalamus, the ventricle was entered through the crevasse. Using the GyroGuide software extension for 3D Slicer (http://download.slicer.org), the angle between the trajectory and each of the three reference planes was automatically calculated. The endoscopic surgery procedure was performed under general anesthesia with the patient in the supine position. A contra-lateral EVD was performed first if the Graeb score of the contra-lateral ventricle was $\geq 2$. Second, a $4-\mathrm{cm}$ incision was made at Kocher's point. A Burr hole was drilled and a 2.5-cm bone flap was milled off. A transcortical puncture was made with an $18-\mathrm{mm}$ rigid plastic rod. A relatively big diameter was able to reduce the frequency of adjustment of the sheath and support the evacuation of hematoma. According to the smartphone-assisted navigation, the puncture depth was the distance between Kocher's point and the target, which was calculated using the 3D Slicer software $(7,12,13)$. When the target was reached, a transparent sheath was placed along the rod. The rod was then removed and the sheath was fixed by a retractor $0^{\circ}$ endoscope (Neuroendoscope; Aesculap, Inc.) and a suction tube was inserted through this transparent sheath, allowing for the removal of hematomas. A slim bipolar cauterizer was used to cauterize the vessel when bleeding. When all procedures were completed, a drainage tube was left in the ventricle in accordance with the hematoma evacuation rate. The EVD procedure after endoscopic surgery was the same as that performed in the EVD group. No urokinase was administered during this procedure. Permanent ventriculo-peritoneal (VP) shunt surgery was performed if patients were diagnosed with hydrocephalus 3 weeks after surgery and total protein in the CSF was $<0.45 \mathrm{~g} / 1$.

Outcome evaluation. The pre-operative characteristics recorded included the pre-operative GCS score, age, ICH volume and severity of IVH. A simplified equation was used to estimate the volume of hematoma: $1 / 2 \mathrm{~A} \times \mathrm{B} \times \mathrm{C}$, where $\mathrm{A}$ is the maximum width measured, $\mathrm{B}$ is the length and $\mathrm{Cis}$ the height (14). The Graeb score was used to evaluate the severity of IVH, which is the sum of the score in each ventricle; 4 is the maximum score in each lateral ventricle and 2 is the maximum score in the third and fourth ventricles (15). The modified Rankin Scale (mRS) (16) was used to evaluate the prognosis of patients. The mRS includes seven grades: 0 , no symptoms; 1, no significant disability; 2, slight disability; 3 , moderate disability; 4, moderately severe disability; 5 , severe disability; and 6, death. All patients were followed up at 180 days after ictus using the mRS by telephone or at the clinic. A favorable outcome was defined as a 180 -day $\mathrm{mRS}$ score $\leq 3$, whereas an unfavorable outcome was defined as a 180-day mRS score $>3$.

Statistical analysis. All statistical analyses were performed using SPSS 19.0 (IBM Corp.). An unpaired t-test or $\chi^{2}$ test was used for comparison between groups, as appropriate. Normally distributed data are expressed as the mean \pm standard deviation and were compared using an unpaired t-test. $\mathrm{P}<0.05$ was considered to indicate a statistically significant difference.

\section{Results}

Patient characteristics. From January 2016 to August 2017, 64 patients diagnosed with TH were treated at the Departments of Neurosurgery and NICU, Central Hospital of Jinzhou (Jinzhou, China). Patients with IVH caused by $\mathrm{TH}$ who had a GCS $\leq 12$ were indicated for surgery. Finally, 40 patients were included in the present study, with 20 patients allocated to the EVD group and 20 to the endoscopic surgery group. The clinical characteristics of the patients in each group are presented in Table I. The average age of the patients was $59.9 \pm 8.7(42-73)$ years in the EVD group and 61.0 \pm 8.5 (48-70) years in the endoscopic group $(\mathrm{P}>0.05)$. The mean pre-operative GCS score was 7.7 \pm 1.7 (5-10) for the EVD group and $7.4 \pm 2.3$ (4-10) for the endoscopic group $(\mathrm{P}>0.05)$. In the EVD group, the mean hematoma volume was $34.8 \mathrm{ml}$, and in the endoscopic group, it was $35.2 \mathrm{ml}(\mathrm{P}>0.05)$. The baseline data, including age, GCS, hematoma volume and Graeb score, were not significantly different between the endoscopic group and the EVD group.

Clinical outcomes. The clinical outcomes are presented in Table II. The length of NICU stay was $6.4 \pm 4.4$ days in the 
Table I. Clinical data of the patients with intraventricular hemorrhage caused by thalamic hemorrhage.

\begin{tabular}{lccc}
\hline Parameter & EVD $(\mathrm{n}=20)$ & Endoscopic $(\mathrm{n}=20)$ & P-values \\
\hline Age (years) & $59.9 \pm 8.7$ & $61.0 \pm 8.5$ & 0.64 \\
Initial GCS & $7.7 \pm 1.7(5-10)$ & $7.4 \pm 2.3(4-10)$ & 0.64 \\
ICH volume $(\mathrm{ml})$ & $34.8 \pm 8.4(25-50)$ & $35.2 \pm 7.4(25-50)$ & 0.88 \\
Graeb score & $7.5 \pm 2.2(4-10)$ & $7.4 \pm 2.3(4-10)$ & 0.95
\end{tabular}

Values are expressed as the mean \pm standard deviation (range). EVD, external ventricular drainage; GCS, Glasgow Coma Scale; ICH, intracerebral hemorrhage.
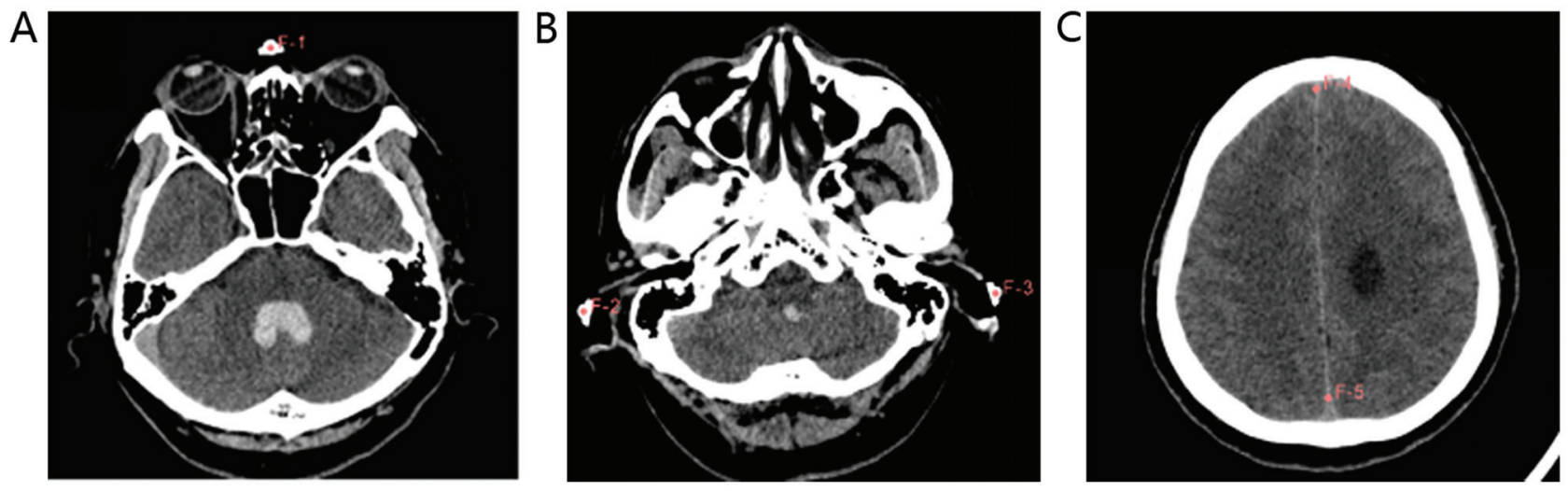

D

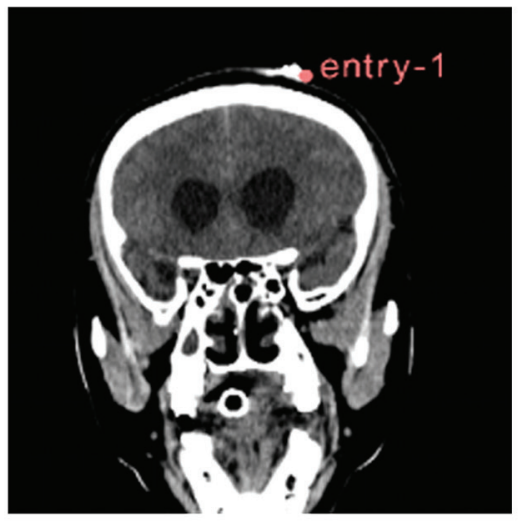

G

\section{$\checkmark$ Calculation}

Angle relation to plane ' $\mathrm{h}---$ Red' $^{2}$ Angle relation to plane 'B---Green' $\underline{14.9}$ Angle relation to plane 'C---Blue' 40.8

Calculation angles
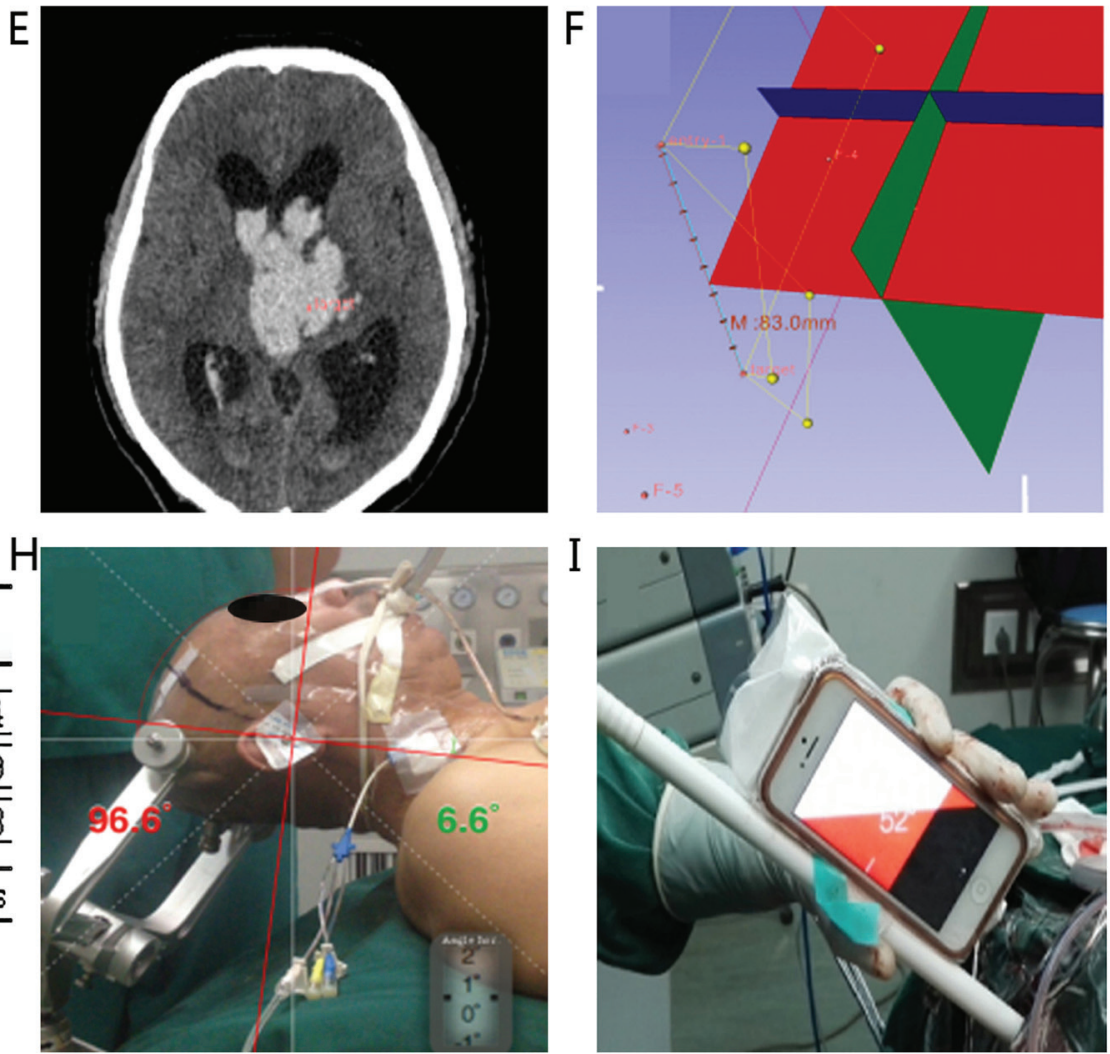

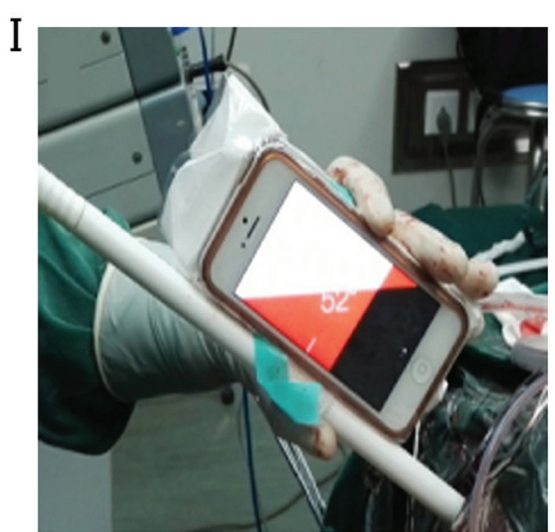

Figure 1. Procedure of smartphone-assisted navigation for endoscopic surgery. Three fiducial markers were attached to the patient's head at different locations: (A) Nasion (F-1); (B) external auditory canal of both sides (F-2 and F-3). (C) F-4 and F-5 were marked on the midline according to the computed tomography image. (D) Entry point (Kocher's point). (E) Target point. (F) Length of trajectory. (G) In the present case, the angle between the trajectory and plane A was $45.4^{\circ}$. (H) The patient's head was strictly fixed and the rotation of reference plane A was measured with an iPhone5S. The line between the nasal pad and the external auditory canal formed plane A. With an iOS app (VirLaser Level 2.5, kab70@ @rambler.ru), the angle between the reference plane A and the horizon was measured as $96.6^{\circ}$. The actual angle between the trajectory and the vertical plane was calculated as follows: $45.4^{\circ}+96.6-90^{\circ}=52^{\circ}$. (I) The iPod touch controlled the direction of the rod. The lever was tilted along the plane until the angle displayed on the iPod touch (iOS app A Level; PosiMotion) until it reached $52^{\circ}$, and then the lever was inserted to a depth of $83 \mathrm{~mm}$ at the target point (13). 
Table II. Clinical outcomes of patients with intraventricular hemorrhage caused by thalamic hemorrhage.

\begin{tabular}{lcccc}
\hline Parameter & EVD $(\mathrm{n}=20)$ & Endoscopic (n=20) & P-value & Odds ratio (95\% CI) \\
\hline Length of NICU stay & $8.4 \pm 4.6$ & $6.4 \pm 4.4$ & 0.17 & ND \\
30-day mortality rate & $2(10 \%)$ & $1(5 \%)$ & 0.55 & ND \\
90-day mortality rate & $4(20 \%)$ & $3(15 \%)$ & 0.68 & ND \\
Postoperative GCS & $10.2 \pm 3.4$ & $11.3 \pm 3.3$ & 0.31 & ND \\
Modified rankin scale & $4.2 \pm 1.4$ & $3.7 \pm 1.2$ & 0.76 & ND \\
VP shunt rate & $10(50 \%)$ & $5(15 \%)$ & 0.02 & $5.7(1.27-25.53)$
\end{tabular}

Values are expressed as the mean \pm standard deviation or $\mathrm{n}(\%)$. CI, confidence interval; EVD, external ventricular drainage; ICH, intracerebral hemorrhage; GCS, Glasgow Coma Scale; VP, ventriculoperitoneal; NICU, neurological intensive care unit; ND, not determined.
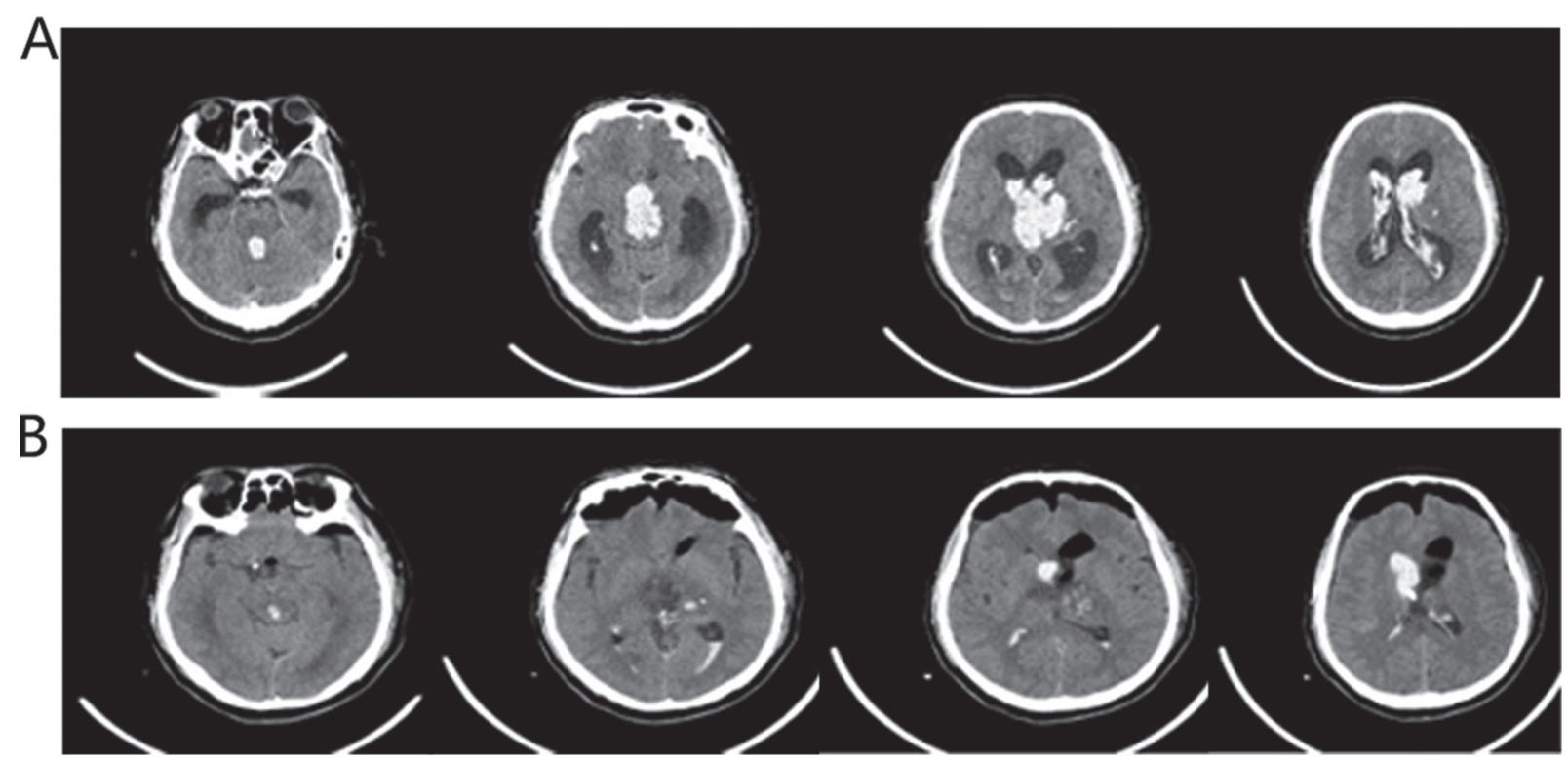

Figure 2. Representative case. (A) Pre-operative CT scan illustrating left-sided thalamic hemorrhage with rupture into the bilateral ventricles and 3rd and 4th ventricles. The patient had developed acute hydrocephalus. (B) Post-operative CT scan illustrating complete removal of the thalamic hematoma, including hematoma of the left-side lateral ventricle and 3rd ventricle. CT, computed tomography.

endoscopic surgery group and $8.4 \pm 4.6$ days in the EVD group; this difference was not statistically significant $(\mathrm{P}>0.05)$. The 30-day and 90-day mortality rates were 5 and $15 \%$ in the endoscopic surgery group, and 10 and $20 \%$ in the EVD group, respectively. However, statistical analysis demonstrated no significant difference due to an insufficient number of cases $(\mathrm{P}>0.05)$. The mean $\mathrm{mRS}$ score was $3.7 \pm 1.2$ in the endoscopic surgery group and $4.2 \pm 1.4$ in the EVD group ( $P>0.05)$. The VP shunt rates were $50.0 \%$ in the EVD group and $15.0 \%$ in the endoscopic surgery group. Patients in the EVD group had a significantly higher VP shunt rate $(\mathrm{P}=0.02$; odds ratio, 5.7) compared with those in the endoscopic surgery group. Endoscopic surgery significantly reduced permanent shunt dependency for IVH caused by $\mathrm{TH}$ compared with EVD alone. No secondary infections were observed in any of the 40 patients.

Case report. A 58-year-old man was transferred to our hospital due to sudden loss of consciousness and vomiting several times. In the emergency room, the patient was in a severe coma (GCS score, 6). A CT scan illustrated left-sided TH with rupture into the bilateral ventricles and the $3 \mathrm{rd}$ and 4 th ventricles. The patient also presented with acute hydrocephalus (Fig. 2). The volume of the hematoma was estimated to be $40 \mathrm{ml}$ according to a simplified equation (12). The patient underwent a contra-lateral EVD and endoscopic surgery to evacuate the thalamic hematoma. A post-operative CT scan demonstrated complete removal of the thalamic hematoma, including hematoma of the left-side lateral ventricle and the 3 rd ventricle (Fig. 2). The right-side ventricular drainage was kept for 5 days after surgery. Intraventricular injection of 20,000 units of urokinase twice a day was applied for 4 days. The patient regained consciousness 2 weeks later and was sent to a rehabilitation center. At 3 months, the patient's mRS score was 3 (Video S1).

\section{Discussion}

Our group previously reported a procedure performed on 8 patients by Kocher's point approach using 3D Slicer software and handheld smartphone-assisted navigation (7), among 
whom only 1 patient required VP shunt surgery. To further demonstrate the feasibility and efficacy of this surgical approach, 40 patients were assigned to an EVD group or an endoscopic surgery group in a non-randomized fashion for the present study. There were no significant differences between the two treatment groups with regard to their 30- or 90-day mortality rate or mRS score at 6 months after the onset of TH. As compared with EVD alone, smartphone-assisted endoscopic surgery via Kocher's point was safe and feasible, and significantly reduced dependency on permanent shunt due to hydrocephalus. In general, there are four types of $\mathrm{TH}$ according to the anatomical location of the hemorrhage: Posterior-lateral, anterior-lateral, medial and dorsal (17). The first two types of hemorrhage may easily rupture into the trigone of the lateral ventricle. This may obstruct normal CSF flow and result in acute hydrocephalus. For ICH, removal of the hematoma is the most important step. This does not only reduce the hematoma volume and edema formation, which leads to reduction in intracranial pressure, but also reduces neurotoxic edema that is caused by increased thrombin and blood degradation products (18). Therefore, it is beneficial to remove the hematoma as soon as possible so as to reduce secondary injuries of $\mathrm{ICH}$, particularly for severe TH accompanied with IVH.

Severe IVH has a high mortality and morbidity rate due to multiple complications, including obstructive hydrocephalus, hypothalamic storm and hyperthermia. A previous study identified that EVD surgery was able to relieve hydrocephalus, but not to prevent shunt-dependent hydrocephalus, as IVH disrupted CSF circulation (16). IVH is a negative prognostic factor in $\mathrm{ICH}$ and permanent shunt dependency is present in a substantial proportion of patients $(6,19)$. Strategies to prevent permanent shunting are increasingly important, as shunt malfunction and other shunt-associated complications occur frequently and are associated with increased morbidity (6). In the present study, 13 out of 40 patients with IVH caused by $\mathrm{TH}$ required VP shunt surgery. The overall VP shunt rate was $32.5 \%$, but only $15 \%$ of patients in the endoscopic surgery group required VP shunt surgery, whereas $50 \%$ of those in the EVD group required shunt surgery. The EVD group had a significantly higher VP shunt rate $(\mathrm{P}=0.02$; odds ratio, 5.7). No endoscopic third ventriculostomy (ETV) was performed in the present study and the possible reason may be that endoscopic surgery was able to evacuate the hematoma while protecting the unaffected areas of the brain.

There are numerous strategies to prevent hydrocephalus $(6,20,21)$. Huttner et al (20) reported that using temporary lumbar drainage was able to reduce VP shunt rates after $\mathrm{ICH}$. In a study published ten years later, the same group recommended lumbar drainage for treating IVH and reducing VP shunt rates (21). Oertel et al (22) explored the value of ETV in the treatment of patients who had suffered IVH with obstructive hydrocephalus. They reported that in the ETV group, which consisted of 33 patients, only two patients who suffered from IVH $(2 / 33,5.9 \%)$ required VP shunt surgery. Therefore, it was proposed that ETV is a safe treatment for IVH-associated obstructive hydrocephalus. Compared with the EVD group, ETV had a lower risk of infection and a low VP shunt rate.

Obaid et al (23) performed a retrospective analysis of 78 consecutive patients who underwent ETV at their institution. They identified 17 consecutive patients who underwent ETV for obstructive hydrocephalus associated with IVH. None of the patients who suffered from IVH and underwent ETV required VP shunt surgery. To treat primary $\mathrm{TH}$ and reduce secondary injury of TH and IVH, certain studies have used minimally invasive surgery to mobilize sedentary blood and enhance CSF clearance through intraventricular fibrinolysis (IVF) $(5,24,25)$. Chen et al (5) performed stereotactic aspiration with subsequent thrombolysis for moderate $\mathrm{TH}$. They concluded that IVF is safe and feasible for post-hemorrhagic hydrocephalus and may markedly reduce the requirement for shunt surgery.

In the present study, endoscopic surgery was performed via Kocher's point by smartphone-assisted navigation to evacuate hematomas of TH and IVH. First, this approach is a modification of the EVD approach and suitable for a minimally invasive strategy. Second, smartphone-assisted navigation was used to ensure the transparent sheath arrived precisely at the target, which reduced unnecessary surgery-induced injury. Modern navigation systems are helpful but expensive. A low-cost solution to locate the hematoma or brain lesion and navigate the surgery under the assistance of augmented reality would be beneficial. The accuracy of smartphone navigation is fine and acceptable (3). Third, the hematoma was removed aggressively by neuroendoscopy, including TH and IVH. During the endoscopic surgery, only the IVH and soft TH were removed, while the hard hematoma was left in the hematoma cavity as described by Chen et al (6). Compared with other approaches, the Kocher's point approach allowed for improved evacuation of hematomas, as the whole lateral ventricle was visible under neuroendoscopy. In the present study, the third ventricular hematoma was evacuated through the foramen of Monro and ETV was possible when required, even though none of the patients of the present study underwent an ETV procedure. Finally, a drainage tube was left in the ventricle to evacuate the bloody CSF and perform IVF to hasten the resolution of IVH. No urokinase was administered during the endoscopic procedure to avoid rebleeding. Intra-ventricular injection of a thrombolytic (20,000 units of urokinase, twice a day) was performed $6 \mathrm{~h}$ after surgery. The urokinase was injected through the catheter to dissolve the residual hematoma and facilitate drainage, and then the drainage bottle was opened but elevated to the height of $27 \mathrm{~cm} \mathrm{H}_{2} \mathrm{O}$ for $2 \mathrm{~h}$ to allow for drug and hematoma interaction. This avoided high intracranial pressure and undetected obstructive hydrocephalus. No secondary infections were observed among the 40 patients in the present study.

The present study demonstrated that endoscopic surgery lowered the shunt-dependent hydrocephalus rate from 50 to $15 \%$ in IVH caused by TH compared with the EVD group. Endoscopic surgery is able to remove primary hematoma and intraventricular blood quickly, and may rapidly reverse ventricular dilatation and reduce intracranial pressure. Developing hydrocephalus may be prevented by removing primary and IVH hematomas and reversing ventricle dilatation. After surgery, urokinase was used to dissolve the residual hematoma and facilitate drainage to enhance CSF clearance, as the volume of intraventricular blood is a key prognostic factor associated with poor outcome in patients with IVH (26). All of these procedures helped to reduce the requirement for VP shunt surgery, and a better prognosis was obtained according 
to the outcomes. Compared with Chen et al (6), a lower VP shunt rate was achieved in the present study, which is likely due the patients receiving IVF.

In the present study, the Graeb scores of the endoscopic group were similar to those in the EVD group, but the VP shunt rate was lower $(\mathrm{P}=0.02)$. The 30 -day mortality rate was $5 \%$ in the endoscopic surgery group and $10 \%$ in the EVD group, while the 90-day mortality rate was $15 \%$ in the endoscopic surgery group and $20 \%$ in the EVD group. However, no statistically significant difference was obtained due to the insufficient sample size. The mortality rate was lower compared with that reported by Steinke et al (27) and Chen et al (5). This demonstrated that the present endoscopic and successive procedure was able to remove IVH faster, reduce ventricular dilatation and re-equilibrate CSF circulation, avoiding persistent hydrocephalus and the requirement for VP shunt surgery, and resulting in a lower mortality rate compared with that obtained in other studies.

Although certain studies have demonstrated that endoscopic surgery offers a better surgical outcome compared with EVD in IVH therapy (28), no significant difference in mortality rates, post-operative GCS scores at 2 weeks and mRS scores at 6 months were obtained between the endoscopic surgery and the EVD group of the present study. These outcomes are similar to those of Chen et al (5). The present study addressed certain limitations of Chen et al (5), but certain limitations still exist. The present study had a non-randomized design and a relatively small sample size; therefore, a randomized study with a larger number of patients is warranted.

In conclusion, the present study demonstrated that endoscopic surgery significantly lowered the rate of VP shunt surgery and may shorten the stay at the NICU in patients with IVH resulting from TH compared with traditional EVD. Smartphone-assisted endoscopic surgery via Kocher's point is feasible and safe, and significantly reduces permanent shunt dependency for IVH caused by TH.

\section{Acknowledgements}

Not applicable.

\section{Funding}

This study was supported by Scientific Technology Research Projects of Jinzhou (grant no. 16B1G37) and the Chinese National Key Research and Development Plan (grant no. 2018YFC1312602).

\section{Availability of data and materials}

The datasets used and/or analyzed during the current study are available from the corresponding author on reasonable request.

\section{Authors' contributions}

$\mathrm{XG}$ and $\mathrm{XX}$ are co-first authors. $\mathrm{XG}, \mathrm{XX}, \mathrm{XY}$ and $\mathrm{XC}$ designed drafted the manuscript. DL and $Y Z$ collected the data. $X G$, $\mathrm{XX}, \mathrm{YZ}$ and YX statistically analyzed the data. All authors read and approved the final manuscript.

\section{Ethics approval and consent to participate}

The current study was approved by the Ethics Committee of Central Hospital of Jinzhou (Jinzhou, China). Written informed consent was obtained from each patient's legally authorized representative.

\section{Patient consent for publication}

Not applicable.

\section{Competing interests}

The authors declare that they have no competing interests.

\section{References}

1. Xu X, Chen X, Li F, Zheng X, Wang Q, Sun G, Zhang J and Xu B: Effectiveness of endoscopic surgery for supratentorial hypertensive intracerebral hemorrhage: A comparison with craniotomy. J Neurosurg 128: 553-559, 2018.

2. Ma L, Hou Y,Zhu R and Chen X: Endoscopic evacuation of basal ganglia hematoma: Surgical technique, outcome, and learning curve. World Neurosurg 101: 57-68, 2017.

3. Hou Y, Ma L, Zhu R and Chen X: iPhone-assisted augmented reality localization of basal ganglia hypertensive hematoma. World Neurosurg 94: 480-492, 2016.

4. Nomura S, Ishihara H, Yoneda H, Shirao S, Shinoyama M and Suzuki M: Neuroendoscopic evacuation of intraventricular hematoma associated with thalamic hemorrhage to shorten the duration of external ventricular drainage. Surg Neurol Int 1: pii: 43, 2010.

5. Chen M, Wang Q, Zhu W, Yin Q, Ma M, Fan X, Li Y, Ni G, Liu C, Liu W, et al: Stereotactic aspiration plus subsequent thrombolysis for moderate thalamic hemorrhage. World Neurosurg 77: 122-129, 2012.

6. Chen CC, Liu CL, Tung YN, Lee HC, Chuang HC, Lin SZ and Cho DY: Endoscopic surgery for intraventricular hemorrhage (IVH) caused by thalamic hemorrhage: Comparisons of endoscopic surgery and external ventricular drainage (EVD) surgery. World Neurosurg 75: 264-268, 2011.

7. Ge X, Chen X, Sun J and Li D: Endoscopic surgery via kocher point approach by simply navigation to treat Intraventricular Hemorrhage (IVH) Caused by Thalamic Hemorrhage. Chin J Nerv Ment Dis 43: 176-179, 2017.

8. Teasdale G and Jennett B: Assessment of coma and impaired consciousness. A practical scale. Lancet 2: 81-84, 1974.

9. Auer LM, Deinsberger W, Niederkorn K, Gell G, Kleinert R, Schneider G, Holzer P, Bone G, Mokry M, Körner E, et al: Endoscopic surgery versus medical treatment for spontaneous intracerebral hematoma: A randomized study. J Neurosurg 70: $530-535,1989$.

10. Teernstra OP, Evers SM, Lodder J, Leffers P, Franke CL and Blaauw G; Multicenter randomized controlled trial (SICHPA): Stereotactic treatment of intracerebral hematoma by means of a plasminogen activator: A multicenter randomized controlled trial (SICHPA). Stroke 34: 968-974, 2003.

11. Dey M, Jaffe J, Stadnik A and Awad IA: External ventricular drainage for intraventricular hemorrhage. Curr Neurol Neurosci Rep 12: 24-33, 2012.

12. Fedorov A,BeichelR, Kalpathy-Cramer J, Finet J, Fillion-Robin JC, Pujol S, Bauer C, Jennings D, Fennessy F, Sonka M, et al: 3D Slicer as an image computing platform for the quantitative imaging network. Magn Reson Imaging 30: 1323-1341, 2012.

13. Chen X, Xu BN and Yu XG: iPod touch-assisted instrumentation of the spine: Is it accurate and reliable? Neurosurgery 6: E734-E736, 2014.

14. Kothari RU, Brott T, Broderick JP, Barsan WG, Sauerbeck LR, Zuccarello $\mathrm{M}$ and Khoury J: The ABCs of measuring intracerebral hemorrhage volumes. Stroke 27: 1304-1305, 1996.

15. Graeb DA, Robertson WD, Lapointe JS, Nugent RA and Harrison PB: Computed tomographic diagnosis of intraventricular hemorrhage. Etiology and prognosis. Radiology 143: 91-96, 1982. 
16. Gupta VP, Garton ALA, Sisti JA, Christophe BR, Lord AS Lewis AK, Frey HP, Claassen J and Connolly ES Jr: Prognosticating functional outcome after intracerebral hemorrhage: The ICHOP score. World Neurosurg 101: 577-583, 2017.

17. Kumral E, Kocaer T, Ertubey NO and Kumral K: Thalamic hemorrhage. A prospective study of 100 patients. Stroke 26: 964-970, 1995

18. Wang WZ, Jiang B, Liu HM, Li D, Lu CZ, Zhao YD and Sander JW: Minimally invasive craniopuncture therapy vs. conservative treatment for spontaneous intracerebral hemorrhage: Results from a randomized clinical trial in China. Int J Stroke 4: 11-16, 2009.

19. Miller C, Tsivgoulis G and Nakaji P: Predictors of ventriculoperitoneal shunting after spontaneous intraparenchymal hemorrhage. Neurocrit Care 8: 235-240, 2008.

20. Huttner HB, Nagel S, Tognoni E, Köhrmann M, Jüttler E, Orakcioglu B, Schellinger PD, Schwab S and Bardutzky J: Intracerebral hemorrhage with severe ventricular involvement: Lumbar drainage for communicating hydrocephalus. Stroke 38 183-187, 2007.

21. Huttner HB and Kuramatsu JB: Current treatment concepts in intracerebral hemorrhage. Med Klin Intensivmed Notfmed 112: 695-702, 2017 (In German).

22. Oertel JM, Mondorf Y, Baldauf J, Schroeder HW and Gaab MR Endoscopic third ventriculostomy for obstructive hydrocephalus due to intracranial hemorrhage with intraventricular extension. J Neurosurg 111: 1119-1126, 2009.
23. Obaid S, Weil AG, Rahme R and Bojanowski MW: Endoscopic third ventriculostomy for obstructive hydrocephalus due to intraventricular hemorrhage. J Neurol Surg A Cent Eur Neurosurg 76: 99-111, 2015

24. Morgan T, Awad I, Keyl P, Lane K and Hanley D: Preliminary report of the clot lysis evaluating accelerated resolution of intraventricular hemorrhage (CLEAR-IVH) clinical trial. Acta Neurochir Suppl 105: 217-220, 2008

25. Morgan T,Zuccarello M, Narayan R, Keyl P,Lane K and Hanley D: Preliminary findings of the minimally-invasive surgery plus rtPA for intracerebral hemorrhage evacuation (MISTIE) clinical trial. Acta Neurochir Suppl 105: 147-151, 2008.

26. Nishikawa T,Ueba T,Kajiwara M, Miyamatsu N and Yamashita K: A priority treatment of the intraventricular hemorrhage (IVH) should be performed in the patients suffering intracerebral hemorrhage with large IVH. Clin Neurol Neurosurg 111: 450-453, 2009.

27. Steinke W, Sacco RL, Mohr JP, Foulkes MA, Tatemichi TK, Wolf PA, Price TR and Hier DB: Thalamic stroke. Presentation and prognosis of infarcts and hemorrhages. Arch Neurol 49: 703-710, 1992.

28. Zhang Z, Li X, Liu Y, Shao Y, Xu S and Yang Y: Application of neuroendoscopy in the treatment of intraventricular hemorrhage. Cerebrovasc Dis 24: 91-96, 2007. 